\title{
The Demographics of Hip Dysplasia in the Maine Coon Cat
}

\author{
Randall T. Loder, MD* \\ Rory J. Todhunter, BVSc, MS, $\mathrm{PhD} \dagger$ \\ *Department of Orthopaedic Surgery, Indiana University School of Medicine and \\ James Whitcomb Riley Children's Hospital, Indianapolis, Indiana, 46202 USA, \\ rloder@iupui.edu
}

$\uparrow$ Department of Clinical Sciences, College of Veterinary Medicine, Cornell University, Ithaca, New York, 14853-6401, USA, rjt2@,cornell.edu

This is the author's manuscript of the article published in final edited form as:

Loder, R. T., \& Todhunter, R. J. (2018). Demographics of hip dysplasia in the Maine Coon cat. Journal of Feline Medicine and Surgery, 20(4), 302-307. https://doi.org/10.1177/1098612X17705554 


\begin{abstract}
Objectives: To study the demographics of feline hip dysplasia (FHD) in the Maine Coon Cat.

Methods: The complete hip dysplasia registry (public and private) collected by the Orthopaedic Foundation for Animals through April 2015 was accessed. There were 2,732 unique felines; 2,708 (99.1\%) were Maine Coon cats; only the Maine Coon cats were studied. Variables analyzed were sex, month/season of birth, and hip dysplasia score. Two groups were created: those with and without FHD. A P $<0.05$ was considered statistically significant.
\end{abstract}

Results: The youngest cat with FHD was 4 months of age. The majority of the radiographs $(2,604 / 2,708$ - 96.2\%) were performed between 4 and 60 months of age. Non-borderline scores for these 2,604 cats were available in 2,548, and are the data for this study. The overall prevalence of FHD was $24.9 \%(635 / 2,548)$, and was slightly higher in males $(279 / 1,023-27.3 \%)$ than females $(356 / 1,525-23.3 \%)(P=0.025)$. Those with more severe dysplasia were older. The percentage of bilateral FHD was 56\%, and bilateral cases had more severe dysplasia than unilateral cases but with no age difference. Month/ season of birth nor geographic region of origin did not influence the prevalence of FHD.

Conclusions and Relevance: This is the largest demographic study of FHD in the Maine Coon cat. The overall prevalence in the Orthopaedic Foundation for Animals registry was $24.9 \%$, and slightly higher in males $(27.3 \%)$ than females $(23.3 \%)$. Dysplasia was more severe in bilateral than unilateral cases and with increasing age. Caution should be used when extrapolating these findings to other feline breeds or other groups of Maine Coon cats. Further studies need to be performed amongst other breeds and geographic locations to better understand the demographics of feline hip dysplasia.

Key words: feline, hip dysplasia, Maine Coon cat, demographics, laterality 


\section{Introduction}

Hip dysplasia is a very well known condition in canines ${ }^{1-4}$, especially amongst

certain breeds. There are only a few studies of feline hip dysplasia (FHD) ${ }^{5-10}$ and there has been no study of the demographics of FHD using a large data set. We wished to pursue this question using the Orthopaedic Foundation for Animals (OFA) registry. Upon reviewing the felines from this data set, the vast majority were Maine Coon cats; we are reporting our findings for this breed

\section{Materials and methods}

The data for this study was the complete hip dysplasia registry (both public and private) collected by the Orthopaedic Foundation for Animals (OFA) through April 2015. There were a total of 1,430,979 records. Duplicate records and canine cases were deleted, resulting in 2,732 unique felines; 2,708 were Maine Coon cats and the remaining 24 various other breeds. Only the 2,708 Maine Coon cats were analyzed in this study.

The variables analyzed were sex, month/season of birth, and hip dysplasia score. The OFA hip score uses the American Veterinary Medical Association grading system: 1 = excellent, $2=$ good, $3=$ fair, $4=$ borderline dysplasia, $5=$ mild dysplasia, $6=$ moderate dysplasia and $7=$ severe dysplasia. The borderline score of 4 was excluded creating two groups: those with FHD (scores 5-7) and those without FHD (scores 1-3). Season of birth was arbitrarily defined as winter - December through February, spring - March through May, summer - June through August, and autumn - September through November. For those cats with FHD, laterality was also studied.

\section{Statistical Analyses}

Categorical variables are expressed as frequencies and percentages, and continuous variables as the average \pm 1 standard deviation. Differences between categorical variables were assessed using the Fishers's exact test for 2 × 2 tables and the Pearson's $\chi^{2}$ for tables larger than $2 \times 2$. Differences for continuous variables were assessed with non parametric tests due to non-normal distributions (Mann-Whitney U test for 2 variables, Kruskal Wallis test for $>2$ variables). While the American Veterinary Medical Association grading system is a numerical grade, it is not a continuous variable such as the Norberg angle, but rather a categorical ordinal variable determined by 
subjective criteria (www.offa-org - Hip Dysplasia - OFA X-ray Procedures). For this reason, the hip score was analyzed as a categorical variable. All statistical analyses were performed with Systat 10 software (Chicago, IL, 2000), and a $\mathrm{P}<0.05$ was considered statistically significant.

\section{Results}

The vast majority $(2,604 / 2,732-96.2 \%)$ of the radiographs were performed between 4 and 60 months of age (Figure 1); the youngest cat with FHD was 4 months of age. The hip score in these 2,604 cats was 1 in 114, 2 in 1,240, 3 in 559, 4 in 56, 5 in 426, 6 in 168, and 7 in 41 . Thus, 2,548 cats had non-borderline scores $(<4$ or $>4)$ and comprise the data for this study. The geographic residence for these 2,548 cats was known in 2,450, and was North America in 1,281 (52.3\%), Europe in 1,114 (45.5\%), and Asia, Australia or New Zealand in 55 (2.2\%). The five countries submitting the most number of radiographs were the USA $(1,159-47.3 \%)$, Finland $(249-10.2 \%)$, Sweden (181-7.4\%), Germany (174 - 7.1\%), and Austria (154-6.3\%), accounting for $78.3 \%$ of all cases.

There were 1,525 female and 1,023 male cats (Table 1). The prevalence of FHD was $24.9 \%(635 / 2,548)$, and was slightly higher in males $(279 / 1,023-27.3 \%)$ than females $(356 / 1,525-23.3 \%)(\mathrm{P}=0.025)$. Those without FHD were statistically younger $(19.4 \pm 11.9$ months $)$ than those with FHD $(20.4 \pm 11.6$ months $)(P=0.003)$. Cats with more severe dysplasia were older (Figure 2). The percentage of cats with FHD by age groups is shown in Figure 3. There were no differences in FHD prevalence by month or season of birth.

Bilateral FHD was present in 55.3\% (225/407) of females and 57.5\% (131/228) of males $(\mathrm{P}=0.62)$. The dysplasia was more severe with bilateral involvement (Figure 4$)$. There was no age difference between those with unilateral $(18.9 \pm 11.0$ months $)$ or bilateral $(20.5 \pm 11.7$ months $)$ FHD $(\mathrm{P}=0.07)$. In unilateral cases, the right hip was involved in $126(60.9 \%)$ and the left in $81(39.1 \%)$. There was no difference in the proportion of right and left hips by gender $(\mathrm{P}=0.77)$ or hip score $(\mathrm{P}=0.13)$. There were no differences in the prevalence of FHD between the three geographic regions (North America, Europe, and Asia/Australia/New Zealand), or the USA and Finland, the two countries submitting the largest number of radiographs to the OFA (Table 2). 


\section{Discussion}

The presentation of FHD is gradual with subtle behavioral changes such as inactivity or aggression, lack of response to human attention, reluctance to go up or down stairs, and resistance to handling 7,11 . The onset typically occurs between three months and 3.5 years of age ${ }^{7}$. Physical examination may demonstrate muscle atrophy and restricted hip motion; crepitus or pain on manipulation may occur in the more advanced

case ${ }^{11}$. An Ortolani sign may be present in some cases ${ }^{12}$. The ventrodorsal radiograph is obtained and the Norberg angle measured, with a lower angle reflecting increasing subluxation. The distraction index can also be measured; a higher distraction index indicates more laxity. It should be remembered that cats have shallower acetabulae and more hip laxity than canines $7,13,14$. The diagnosis of FHD is confirmed by noting the shallow acetabulum, subluxation of the femoral head, and often early degenerative changes at the cranial border of the acetabulum 11,13 .

The diagnosis of FHD in the OFA data base was determined using the standard method of a consensus score amongst three different radiologists. The overall accuracy of this distinction between animals with and without hip dysplasia using the OFA scoring schemes was $94.9 \%$ in 1.8 million radiographs (http://www.ofa.org/hd grades.html). Although the system is primarily used in canines, the OFA hip score has been used in a previous study of FHD ${ }^{5}$, and a similar OFA like system was used by the Pennsylvania group ${ }^{14}$. We believe that the consensus amongst three different radiologists gives assurance as to the correctness of the diagnosis. Those cats designated as having FHD likely did have it; and those designated as not having FHD likely did not have FHD. There is a possibility of a false negative diagnosis, if FHD later appeared in a cat as it aged after the radiograph had been submitted. This would only result in a higher prevalence of FHD.

The first mention of FHD appears to be by Peiffer ${ }^{15}$ in 1974 and Holt ${ }^{8}$ in 1978. Peiffer et al described pectineus tenotomy as a treatment but gave no details regarding cases of FHD. Holt described FHD in a three year six month old castrated male Persian cat. Since then there have been three studies discussing FHD aside from case reports or a 
single litter. Köppel ${ }^{9}$ in 1989 at the University of Vienna described 293 cats, of which 285 were Domestic Shorthairs. The prevalence of FHD was $43.4 \%$ in the males $(23 / 159)$ and $56.6 \%$ in the females $(30 / 134)$; this difference by sex is not statistically significant. Langenbach ${ }^{14}$ in 1998 at the University of Pennsylvania studied 78 cats (gender not mentioned) with a $32 \%$ prevalence of FHD. These 78 cats were from 9 different breeds and at least 6 months old when the radiographs were performed; there were no excellent scores even in those without FHD. Keller ${ }^{5}$ in 1999 at the University of Missouri studied 684 cats with, 603 being Domestic Shorthair cats. The overall prevalence of FHD was $6.6 \%$ and did not differ by gender.

This is the largest series to date describing the demographics with FHD. Due to the preponderance of the Maine Coon cat breed in the OFA registry we could only study the demographics of FHD in one breed. Of the 2,732 cats in the registry 2,708 were Maine Coon (99.1\%). The remaining 24 cats were comprised of 10 British Shorthair, six Persian, three Siamese, two Domestic Shorthair, and one Somali, Ragamuffin, and Bengal Tiger. In this study of the Maine Coon cat in the OFA data base the overall prevalence of FHD was $24.9 \%$. There are certain limitations to the findings in this study. First, it only describes the Maine Coon cat population within the OFA data base, which likely has a selection bias, since the OFA registry only consists of those cats whose radiographs were voluntarily submitted for analysis. It is very possible that owners or breeders with obviously dysplastic cats never submitted radiographs. Such a bias in the OFA data set has been previously discussed in canines 16 . Thus the prevalence of $24.9 \%$ is likely a low estimate. A more accurate number would require obtaining radiographs of a large number of Maine Coon cats without any pre-selection by the owners/breeders. Finally, if the cats whose radiographs were performed under 1 year of age underwent repeat radiographic examination after 1 year of age, it is possible that some of the normal cats would have developed FHD, again resulting in a higher prevalence. We chose 4 months of age as the cutoff as that was the age of the youngest cat in the registry having a score of 5-7.

The reason why the OFA data base is comprised of nearly all Maine Coon cats is due to previous reports of hip dysplasia as a specific problem in that breed ${ }^{5}$. The goal of 
our study was to describe the demographics of feline hip dysplasia using presently available data. In the USA, the submission of images to either the OFA registry or others is voluntary as there is no compulsory registry for feline hip dysplasia in North America. Besides the OFA registry, there is another voluntary and public one for the Maine Coon cat. This registry is administered by PawPeds (https://www.pawpeds.com/healthprogrammes/hd.html) and a single radiologist reviews all the radiographs. Other breed clubs are encouraged to join. The most recent data from that registry gives an overall prevalence of FHD in Maine Coon cats of 32.4\% 969/2,988) 17

The $24.9 \%$ prevalence of FHD in this study is similar to that of Langenbach ${ }^{14}$, higher than that of Keller ${ }^{5}$, and lower than that of Köppel ${ }^{9}$. There are several possible explanations for these differences. One possible explanation is that this study involved only Maine Coon cats, while two of the three studies mentioned above are predominantly Domestic Shorthair cats 5,9 with the third a mixture of breeds ${ }^{14}$. This may be due to genetic differences for FHD by breed. Another explanation is that the gene pool for FHD may vary by geographic residence location of the cat. However, we noted no difference in the prevalence of FHD by geographic region. A final explanation may involve the effect of neutering. None of the studies investigated this potential factor, and the status of neutering is not known in the OFA data base. Finally, the $29.4 \%$ prevalence of FDH in the OFA Maine Coon cat database is very similar to the $32.4 \%$ prevalence in the Swedish PawPeds registry 17 . The PawPeds registry uses a four level grading protocol: normal, grade 1 (mild dysplasia), grade 2 (moderate dysplasia), and grade 3 (bad dysplasia). The exact criteria for the dysplastic grades are not given on their web site 17 and are likely different than the OFA American Veterinary Medical Association grading system; however both demonstrate a similar prevalence of FHD.

\section{Conclusion}

This study gives the clinician new, detailed background data regarding the demographics of FHD in the Maine Coon cat. The overall prevalence of FHD in the OFA data base was $24.9 \%$ and slightly higher in males $(27.3 \%)$ than females $(23.3 \%)$. The percentage of bilateral FHD was $56 \%$ (of the $24.9 \%$ ) with no difference by gender. 
The dysplasia was more severe in bilateral cases. Caution should be used when extrapolating these findings to other feline breeds, as well as to other groups of Maine Coon cats. Further studies need to be performed amongst other breeds and geographic locations to better understand the demographics of feline hip dysplasia.

\section{Acknowledgements}

The authors wish to thank Mr. Eddi Dzuik and Jon Curby, Orthopaedic Foundation for Animals, for granting access to the entire hip dysplasia registry.

\section{Funding}

This research was supported in part by the Garceau Professorship Endowment, Indiana University, School of Medicine, Department of Orthopaedic Surgery, and the Rapp Pediatric Orthopaedic Research Endowment, Riley Children's Foundation, Indianapolis, Indiana.

\section{Conflict of Interest}

The authors declare no conflict of interest. 


\section{References}

1 Riser WH. Canine hip dysplasia: cause and control. J Am Vet Med Assoc 1974; 165:360-362.

2 Lust G, Geary JC, Sheffy BE. Development of hip dysplasia in dogs. Am J Vet Res 1973;34:87-91.

3 Lust G, Rendano VT, Summers BA. Canine hip dysplasia: concepts and diagnosis. J Am Vet Med Assoc 1985;187:638-640.

4 Lust G, Williams AJ, Burton-Wurster N, Pijanowski GJ, Beck KA, Rubin G, et al. Joint laxity and its association with hip dysplasia in Labrador retrievers. Am J Vet Res 1993;54:1990-1999.

5 Keller GG, Reed AL, Lattimer JC, Corley EA. Hip dysplasia: a feline population study. Vet Radiol Ultrasound 1999;40:460-464.

6 Patsikas MN, Papazoglou LG, Komninou A, Dessiris AK, Tsimopoulos G. Hip dysplasia in the cat: a report of three cases. J Small Anim Pract 1998;39:290-294.

7 Perry K. Feline hip dysplasia. A challenge to recognise and treat. J Feline Med Surg 2016;18:203-218.

8 Holt PE. Hip dysplasia in a cat. J Small Anim Pract 1978;19:273-276.

9 Köppel E, Ebner J. Die hüftgelenkdysplasie der katze [Hip dysplasia in the cat]. Klientierpraxia 1989;35:281-298.

10 Rabin KL, de Haan JJ, Ackerman N. Hip dysplasia in a litter of domestic shorthair cats. Feline Practice 1994;22:15-18. 
11 Grierson J. Hips, elbows and stifles. Common joint diseases in the cat. J Feline Med Surg 2012;12:23-30.

12 Kerwin S. Orthopedic examination in the cat. J Feline Med Surg 2012;14:6-12.

13 Allan GS. Radiographic features of feline joint diseases. Vet Clin N Am Small Anim Practice 2000;30:281-302.

14 Langenbach A, Giger U, Green P, Rhodes H, Gregor TP, LaFond E, et al. Relationship between degenerative joint disease and hip joint laxity by use of distraction index and Norberg angle measurement in a group of cats. JAVMA 1998;213:1439-1443.

15 Peiffer RL, Young Jr. WO, Blevins WE. Hip dysplasia and pectineus resection in the cat. Feline Practice 1974;4:40-43.

16 Comhaire FH, Snaps F. Comparison of two canine registry databases on the prevalence of hip dysplasia by breed and the relationship with body weight and height. Am J Vet Res 2008;69:330-333.

17 PawPeds. https://pawpeds.com/healthprogrammes/hd.html. Feline hip dysplasia. Accessed February 9, 2017. 


\section{Figure Legends}

Figure 1: Histogram of all 2,708 Maine Coon cats. The vast majority (96.16\%) had the radiographs performed between 4 and 60 months of age.

Figure 2: Average age for each hip score severity. The age difference between the three groups with hip dysplasia (scores 5,6 or 7 ) was statistically significant $(\mathrm{P}=0.00004)$. There were significant differences between the mild and moderate group $(\mathrm{P}=0.0003)$ and the mild and severe group $(\mathrm{P}=0.017)$, but not between the moderate and severe group $(\mathrm{P}=0.99)$.

Figure 3: The number of cats and percentages with and without FHD by different age groups at the time of the radiograph. The actual number of the cats are the values within each section of the bars.

Figure 4: Differences in feline hip dysplasia by severity and unilateral/bilateral nature. The numbers within each box denote the number of cats. The age difference between all three groups was statistically significant $\left(\mathrm{P}<10^{-6}\right)$. There were significant differences between the mild and moderate group $\left(\mathrm{P}<10^{-6}\right)$ and the mild and severe group (PS $=$ $0.0002)$, but not between the moderate and severe group $(\mathrm{P}=0.63)$. 
Table 1: The demographics of hip dysplasia in the Maine Coon cat

\begin{tabular}{|c|c|c|c|c|c|}
\hline & $\begin{array}{l}\text { Without } \\
\text { FHD* }\end{array}$ & With FHD & $\begin{array}{c}\% \text { without } \\
\text { FHD }\end{array}$ & $\begin{array}{c}\text { \% with } \\
\text { FHD }\end{array}$ & $P$ value \\
\hline All & 1913 & 635 & 75.08 & 24.92 & - \\
\hline Age (months) $\dagger$ & $19.4+11.9$ & $20.4+11.6$ & - & - & 0.003 \\
\hline \multicolumn{6}{|l|}{ Sex } \\
\hline $\mathbf{F}$ & 1169 & 356 & 76.66 & 23.34 & 0.025 \\
\hline $\mathbf{M}$ & 744 & 279 & 72.73 & 27.27 & \\
\hline \multicolumn{6}{|l|}{ Month of Birth } \\
\hline Jan & 154 & 55 & 73.68 & 26.32 & 0.83 \\
\hline Feb & 141 & 41 & 77.47 & 22.53 & \\
\hline Mar & 169 & 56 & 75.11 & 24.89 & \\
\hline Apr & 192 & 65 & 74.71 & 25.29 & \\
\hline May & 190 & 61 & 75.70 & 24.30 & \\
\hline Jun & 155 & 51 & 75.24 & 24.76 & \\
\hline Jul & 175 & 48 & 78.48 & 21.52 & \\
\hline Aug & 145 & 56 & 72.14 & 27.86 & \\
\hline Sep & 147 & 39 & 79.03 & 20.97 & \\
\hline Oct & 162 & 54 & 75.00 & 25.00 & \\
\hline Nov & 148 & 59 & 71.50 & 28.50 & \\
\hline Dec & 135 & 50 & 72.97 & 27.03 & \\
\hline \multicolumn{6}{|l|}{ Season of Birth } \\
\hline Autumn & 457 & 152 & 75.04 & 24.96 & 0.99 \\
\hline Spring & 551 & 182 & 75.17 & 24.83 & \\
\hline Summer & 475 & 155 & 75.40 & 24.60 & \\
\hline Winter & 430 & 146 & 74.65 & 25.35 & \\
\hline
\end{tabular}

* FHD = feline hip dysplasia

$\dagger$ average \pm 1 standard deviation 
Table 2: Prevalence of hip dysplasia in the Maine Coon cat by geographic region

\begin{tabular}{|l|c|c|c|c|c|}
\hline & $\begin{array}{c}\text { Without } \\
\text { FHD* }\end{array}$ & With FHD & $\begin{array}{c}\text { \% without } \\
\text { FHD }\end{array}$ & $\begin{array}{c}\text { \% with } \\
\text { FHD }\end{array}$ & P value \\
\hline North America & 930 & 313 & 74.8 & 25.2 & 0.96 \\
\hline Europe & 827 & 271 & 75.3 & 24.7 & \\
\hline All others & 41 & 13 & 75.9 & 24.1 & \\
\hline \multicolumn{5}{|l|}{} \\
\hline Finland & 175 & 69 & 71.7 & 28.3 & 0.58 \\
\hline USA & 825 & 298 & 73.5 & 26.5 & \\
\hline
\end{tabular}

$*$ FHD = feline hip dysplasia 


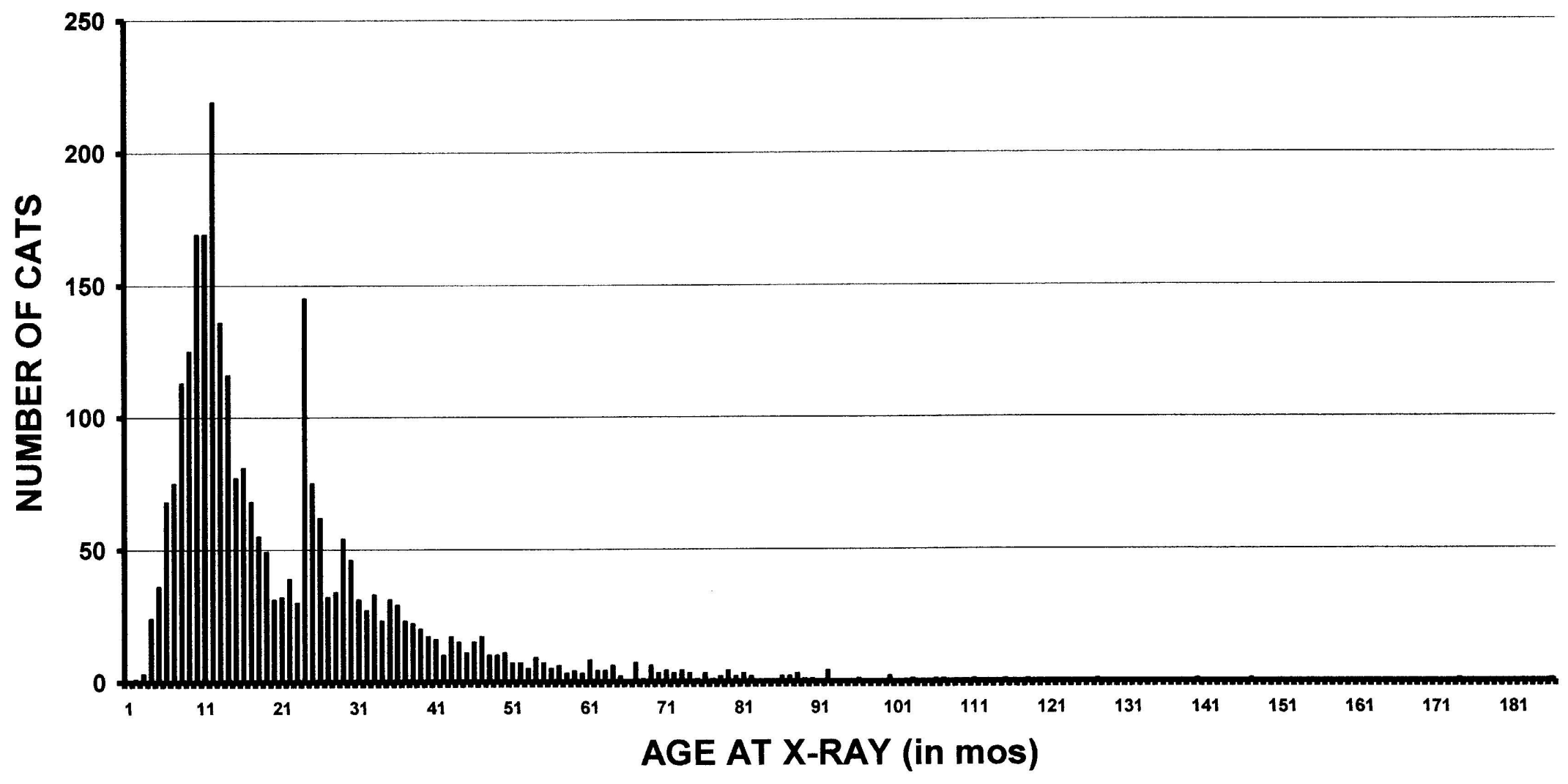




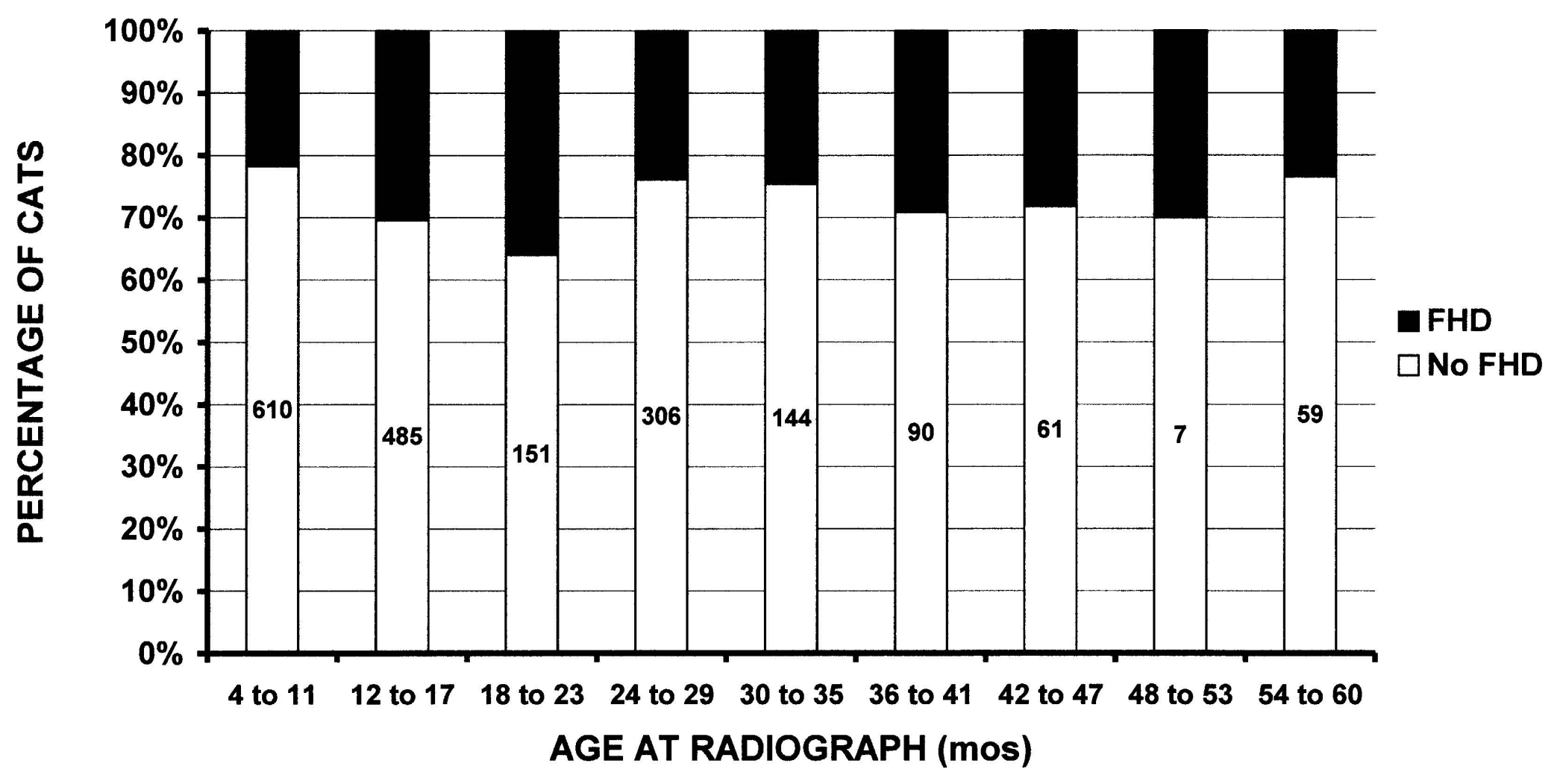




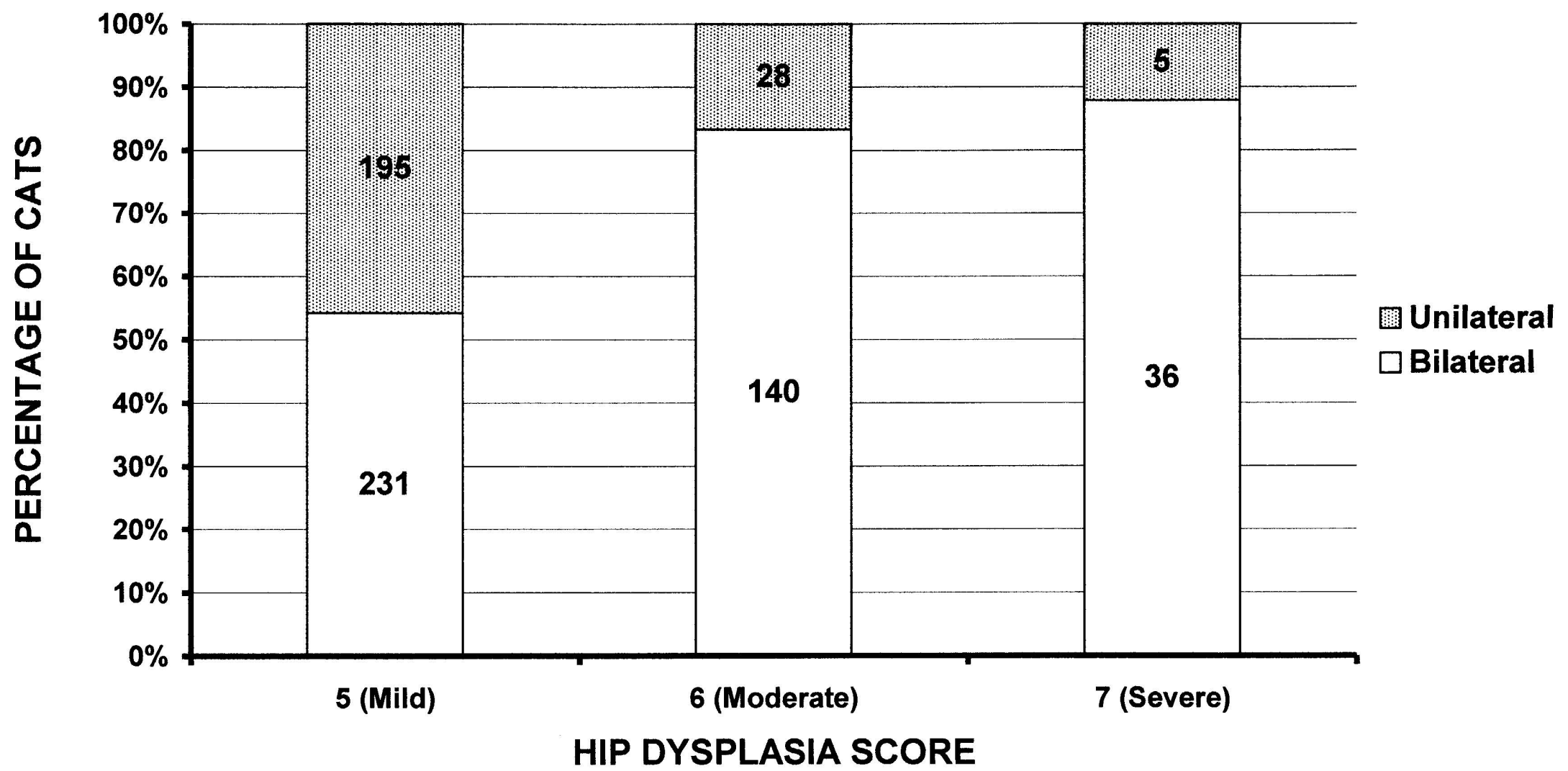

